\title{
Study on the Technology of Leakage Protection
}

\author{
Shen Zixing ${ }^{1, *}$, Shen Quntai ${ }^{1}$, Li Sheng ${ }^{2}$ and Liu Xuelin ${ }^{3}$ \\ ${ }^{1}$ School of Information Science and Engineering, Central South University, Changsha, Hunan, 410083, China \\ ${ }^{2}$ Department of Public Security, Railway Police Collge, Zhengzhou, Henan, 450002, China \\ ${ }^{3}$ Henan Institite of Medical Device Testing, Zhengzhou, Henan, 450003, China
}

\begin{abstract}
Failure leakage of equipment or electric shock may make the residual current amplitude decrease, which causes effective protection of the fault and the existence of dead zone of operation. This paper presents the principle of residual current leakage protection based on vector, using residual current amplitude and residual current variation of fault leakage protection, the realization of mutation leakage, continuous slow change leakage detection and recognition, triangle formulas residual current variation calculation method, orthogonal decomposition calculation method and three-phase equivalent calculation method. Through the analysis of the residual current vector variation, we put forward the corresponding method of delayed electrical leakage protection. According to the residual current amplitude, mutation leakage, slowly varying leakage, electric leakage comprehensive protection, we can reduce the leakage protection action area; improve the reliability of power system.
\end{abstract}

Keywords: Action dead zone, Continuous slow change leakage, RCD.

\section{INTRODUCTION}

Leakage protection is an important protection technology for low voltage electrical protection, are widely used to prevent leakage and prevent accidents of personal electric shock casualties. With the development of electric power, the scope of application of electric leakage protection more and more widely, there are increasingly high requirements. In the TN system, leakage protection is realized by the method of amplitude detection of residual current in formal occasions, so people call it the "residual current operated protective" and not called "leakage protection" [1-4].

With the development of new science and technology, especially the development of intelligent electrical apparatus technology, and provides a broad space for the development of technology of leakage protection. In recent years, people put forward the new theory and new method of leakage protection; promote the development of technology of leakage protection. Some people put forward the protection method of the electric shock of the people according to the shock current waveform, but the distinction and identification of shock current waveform and the general leakage current has the problem, the false action and non action are very high, not the actual application. At the same time, but also the use of amplitude discrimination and phase discrimination technology of leakage protection for personal protection, the correct rate is improved, and is widely applied in the rural power network, but there are still false action, also cannot

*Address correspondence to this author at the School of Information Science and Engineering, Central South University, Changsha, Hunan, 410083, China; E-mail: 35387230@qq.com overcome the action dead zone completely, its application is restricted. At the same time, the electromagnetic leakage protection compatible problems and reliability issues have also been widely studied, and the problem of fault identification and protection has not been a very good solution $[5,6]$. So can not mistake in the normal movement of the right protection of electric leakage in fault leakage, become an important part of our research, and also the development direction of earth leakage protection technology.

In this paper, based on analysis of leakage protection technology of residual current, conducts the research to the fault and the TN grid system in the electric shock, according to the identification of fault current surplus current vector, puts forward the leakage protection theory based on residual current vector. Variation widely application of $[7,8]$ in power system relay protection, while in the field has not been investigated leakage protection. This paper study on the alternating current contactor leakage characteristics of mathematical representation, the establishment of protection system for residual current, leakage, slow change leakage mutation, improve the effectiveness of the earth leakage protection and reliability without increasing the false action and non action of new.

The existence of leakage protection technology based on the problem of residual current amplitude

A residual current of $I_{1}$ in normal operation of the power grid, if at some point in the grid generation of fault equipment (or someone gets an electric shock), the fault current is $I_{2}$, and then the residual current into the synthesis of $I$, see Fig. (1). 


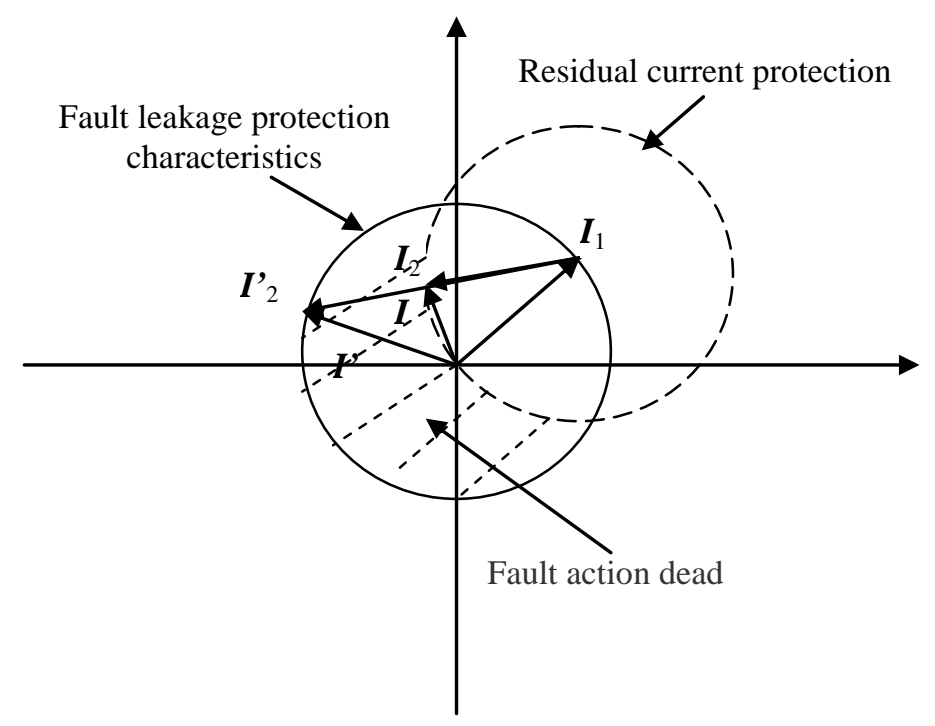

Fig. (1). Schematic diagram of operating dead band of RCD.
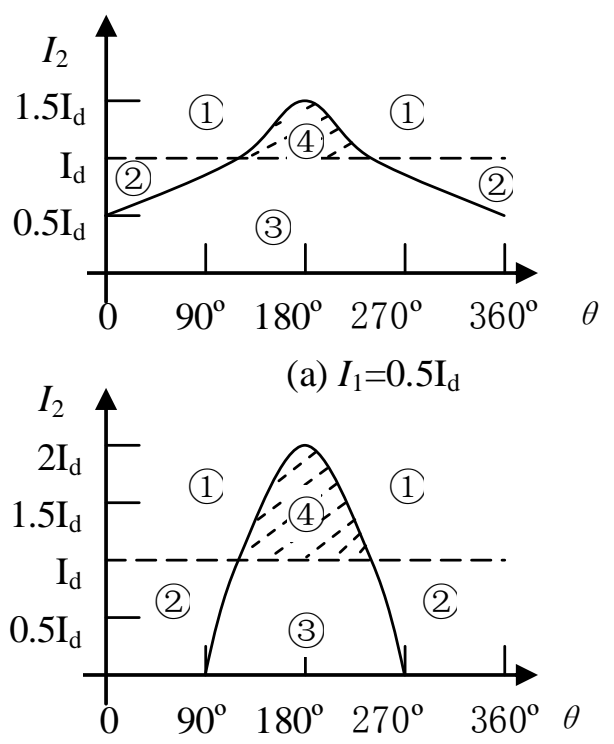

(b) $I_{1}=\mathrm{I}_{\mathrm{d}^{-}}$

Fig. (2). Schematic diagram of operating dead band of RCD.

In Fig. (1), the residual current protection characteristics as no action region Park, park outside the action zone. When there is a residual current $I_{1}$ (less than action value in the residual current protection characteristic Park,), generation of fault current $I_{2}$ (in the fault leakage protection characteristics of vineyard), its value is greater than the action value, should be the fault of leakage protection, but because the transformer can only detect the residual current of I, and I in the remaining residual current protection characteristic Park (far less than action value), so it will not protect. Only the fault current to $\boldsymbol{I}_{2}$ (far more than action value), residual current detection of I' only in the residual current protection characteristics of garden, the protection of the fault current, so Figure 1 dotted shadow part of fault action dead zone.

According to the amplitude of leakage protection, fault current $I_{2}$ satisfy (1) when the leakage protection action.

$$
I_{2} ! \mathrm{Y} I_{d}\left(\sqrt{1 K^{2} \sin ^{2} \theta} K \cos \theta\right)
$$

In the formula : $K=I_{1} / I_{\mathrm{d}}, \quad \theta$ is the phase angle difference of $\mathrm{I} 2$ and $I_{1}$.

From equation (1) can be seen, generating a fault leakage power system without residual current, using amplitude method can correct protection on fault leakage. And the existence of residual current in the power grid, fault leakage protection features not only the amplitude and fault related, also and its phase angle on. The existence of residual current, fault leakage protection characteristic curve has been shown in Fig. (2).

Fig. (2a) is a fault residual current operating characteristics of $I_{1}=0.5 \mathrm{I}_{\mathrm{d}}$ cases already exists, the upper for the fault leakage action area, the solid line. In the fault movement in 
the area, although the fault current is the part of less than leakage protection action value, but the remainder current amplitude at this time is larger than the leakage protection action value $I_{d}$, So the leakage protection action. In the fault does not act in the area, although the fault current is greater than the fourth part of the leakage protection action value, but because of the residual current less than leakage protection action value, so no action, for the action dead zone of fault, Fault action dead zone change along with the change of residual current $I_{1}$ already exists in the power grid [9-11].

Residual current $I_{1}$ already exists in the power grid near the leakage protection action value, the maximum fault leakage action generated, as shown in Fig. (2b), the maximum fault leakage action value reached $2 \mathrm{I}_{\mathrm{d}}$.

Fig. (1) and Fig. (2) is the same, in Fig. (1), the residual current of different $\boldsymbol{I}_{1}$ in polar coordinates position, leakage protection of regional fault is different, but the characteristics of residual current protection are based on $\boldsymbol{I}_{1}$ as a circle. When the residual current of $\boldsymbol{I}_{1}$ is zero, the characteristics of fault leakage protection and residual current protection characteristics of coincidence, no fault leakage action dead zone; when the residual current of $\boldsymbol{I}_{1}$ close to the residual current, fault action dead zone maximum, and when the residual current and fault current of $\boldsymbol{I}_{1}$ and $\boldsymbol{I}_{2}$ reverse phase, the action current of up to 2 times the maximum. Action value

\section{CALCULATON MODEL}

\subsection{Fault Recognition Technology}

In the residual current protection technology, basis, people generally use the residual current amplitude as earth leakage protection however, leakage current in power system is not only the amplitude of the branch, and the phase angle difference. In the power network and electrical equipment when in normal work, the existence of the normal leakage current, and the normal leakage current due to the influence of various factors.

There is leakage current under the normal conditions, when the grid or electric equipment from fault resulting in leakage current, will inevitably lead to the change of residual current, and the change of residual current is not only the amplitude variation, and the change of the phase angle. Therefore, identification of fault can be achieved by changing the quantity of residual current $[12,13]$.

The power supply to the Zhou Bo for the detection of residual current detection unit, the data is: $\dot{I}_{0}, \dot{I}_{1}, \dot{I}_{2}, \ldots$, $\dot{I}_{i}, \ldots$ Residual current changes the amount of any I time can be described by the formula (2) said.

$\dot{I}_{\Delta i}=\dot{I}_{i} \dot{I}_{m}$

In the formula, $\dot{I}_{\Delta i}$ is Changes of amount of $\mathrm{i}$ residual current moment, $\dot{I}_{i}$ is the moment of I residual current, $\dot{I}_{m}$ for the residual current I moment ago m moment.

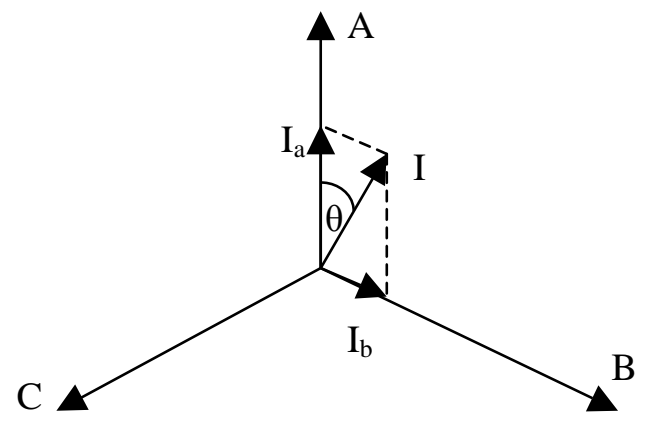

Fig. (3). Schematic diagram of leakage current calculation method.

Fault in arbitrary I time by changing the amount of residual current time to indirectly express.

\subsection{The Calculation Method of Triangle Formula Varia- tion of Residual Current Vector}

The choice of a reference phase, phase generally chooses A phase voltage as the reference phase, as shown in Fig. (3), for the detection of residual current. Can detect the amplitude of the residual current of I, but also can detect the phase angle $\theta$.

In the residual current detection $\dot{I_{m}}$ for $\mathrm{M}$ moment, and its amplitude is $I_{m}, \theta_{m}$ argument. In the residual current detection $\dot{I}_{i}$ for i moment, and its amplitude is $I_{i}, \theta_{i}$ argument. According to the calculation formula of trigonometric function, can calculate the residual current variation.

$$
\begin{aligned}
& I_{\Delta \mathrm{r}}=\sqrt{I_{m}^{2}+I_{i}^{2}+2 I_{m} I_{i} \cos \left(\theta_{m} \theta_{i}\right)} \\
& \theta_{\Delta}=\operatorname{arctg} \frac{I_{m} \sin \theta_{m} I_{i} \sin \theta_{i}}{I_{m} \cos \theta_{m} I_{i} \cos \theta_{i}}
\end{aligned}
$$

Triangle formula calculation method, but the calculation is not simple, because it involves the calculation of trigonometric functions, not easy to write MCU program.

\subsection{The Calculation Method of Orthogonal Variation of Residual Current Vector Decomposition}

The residual current is decomposed into two orthogonal current component, can be calculated by formula (5) orthogonal component of residual current.

$$
\left\{\begin{array}{c}
I_{\mathrm{x}}=I \cos \theta \\
I_{\mathrm{y}}=I \sin \theta
\end{array}\right.
$$

To detect residual current i moment and $\mathrm{M}$ moment, by type (5) are calculated by the orthogonal component of current between the orthogonal component of current direct subtraction obtained after orthogonal components of volume change. Variation design for orthogonal components $I_{\Delta x}$ and $I_{\Delta y}$, may through the type (6) and (7) the calculation of 
fault current is the moment of a new generation, which is to calculate the residual current variation.

$I_{\Delta \mathrm{r}}=\sqrt{I_{\Delta \mathrm{x}}^{2}+I_{\Delta \mathrm{y}}^{2}}$

$\theta_{\Delta}=\operatorname{arctg} \frac{I_{\Delta \mathrm{y}}}{I_{\Delta \mathrm{x}}}$

Can be achieved through integral (5) calculations, MCU is programmed through the addition can be realized, to avoid the computation of trigonometric function, simplified the programming of MCU program.

\subsection{The Calculation Method of Equivalent Variation of Three-phase Residual Current Vector}

The residual current is obtained by the superposition of leakage current of each phase, will be calculated for each phase the leakage current, which not only simplifies the calculation of residual current variation, also can acquire the leakage distribution grid. In a phase A phase voltage as the reference phase, to detect the residual current amplitude and phase angle. When the $\mathrm{C}$ phase residual current of Ic is zero, according to the $I$ and $\theta$, can get the A phase and $B$ phase residual current of $\mathrm{Ia}$ and $\mathrm{Ib}$, so as to realize the detection of leakage current of each phase. When the three-phase have leakage, leakage current detect is minimum phase offset the leakage current, the residual current of other two phases unbalance.

When the residual current detected at $\mathrm{A}$ phase and $\mathrm{B}$ phase, $\mathrm{C}$ phase residual current minimum offset to 0 , by type (8) of all phase equivalent residual current is calculated.

$$
\left\{\begin{array}{l}
I_{a}=I\left(\cos \theta+\frac{\sin \theta}{\sqrt{3}}\right) \\
I_{b}=I \frac{\sin \theta}{\sqrt{3} / 2} \quad\left(0^{\circ} \leq \theta<120^{\circ}\right) \\
I_{c}=0
\end{array}\right.
$$

When the residual current detected at $\mathrm{B}$ phase and $\mathrm{C}$ phase offset between, Ia is 0 . When the residual current detected at $\mathrm{C}$ phase and $\mathrm{A}$ phase offset between, $\mathrm{Ib}$ is 0 as of computational method of the two cases (8) as proposed.

If the change of three-phase equivalent residual current at the same time, namely the A phase variation is $I_{\Delta a}$, B phase change amount is $I_{\Delta \mathrm{b}}, \mathrm{C}$ phase change amount is $I_{\Delta \mathrm{c}}$, when $I_{\Delta \mathrm{c}}$ is the minimum value for the current change, the remaining amount of the moment can type (9) and (10) computing.

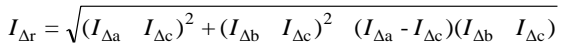

$$
\begin{aligned}
& \theta_{\Delta}=\operatorname{arctg} \frac{\sqrt{3}\left(I_{\Delta \mathrm{b}} I_{\Delta \mathrm{c}}\right) / 2}{\left(I_{\Delta \mathrm{a}}-I_{\Delta \mathrm{c}}\right)-\left(I_{\Delta \mathrm{b}} I_{\Delta \mathrm{c}}\right) / 2} \quad\left(0^{\circ} \leq \theta_{\Delta} \leq 120^{\circ}\right)
\end{aligned}
$$

When the power grid and electrical equipment on the ground impedance of non resistance, although the residual current calculation does not represent the residual current of each phase, but the residual current variations calculated is completely correct, still can achieve the detection of the leakage current of the new generation.

After the mathematical transform and integral, MCU is programmed by additive also can realize the type (8) calculation, but also can obtain the equivalent current in each phase of.

\section{LEAKAGE PROTECTION MODEL OF RESIDUAL CURRENT VECTOR VARIATION METHOD}

\subsection{Leakage Protection Model Based on Residual Cur- rent Vector Variation}

Proposed model for the type (11) has been set residual current and its change rate of leakage protection, realization of leakage action without dead zones:

$$
\begin{gathered}
\left|\dot{I_{i}}\right| \geqslant \mathrm{I}_{\mathrm{d}} \\
\left|\dot{I}_{\Delta i}\right| \geqslant \mathrm{I}_{\mathrm{d}}
\end{gathered}
$$

According to equation (11) exists leakage protection, leakage protection characteristics of residual current I1 cases which have been shown in Fig. (4).

In Fig. (4), the $\boldsymbol{I}_{1}$ fault zone is different, no action is different. When $\boldsymbol{I}_{1}$ is 0 , Residual current protection characteristics and fault leakage protection characteristics coincide completely. Residual current action value and changes in the amount of action value is set to the same value, whether it is the residual current action exceed the value, or change in excess of action value, must be the leakage current line or equipment more than action value. In this case, not only will not produce false action area, also won't produce refused action area.

In Fig. (2), the zone of action into 1, 2, 3 area, 3 for the no action region, to achieve the right protection of fault, overcomes the action dead zone.

Residual current amplitude value of the action and residual current variation law of value can be set to different values of action. If the residual current variation of the action value is small, reduce the leakage is not action area, but prone to error action; if the residual current variation of the action value, increase the creep age no action region, disoperation will reduce, but prone to reject action. When the residual current variation of the action value is infinity, leakage is not any action region of residual current action area, therefore a special case of leakage protection model in this paper, the traditional method of residual current protection of the proposed. In addition, you can also limit angle on residual current variation, when a phase the same phase and threephase power residual current variation in, if the residual current variation amplitude greater than action value, protection, 


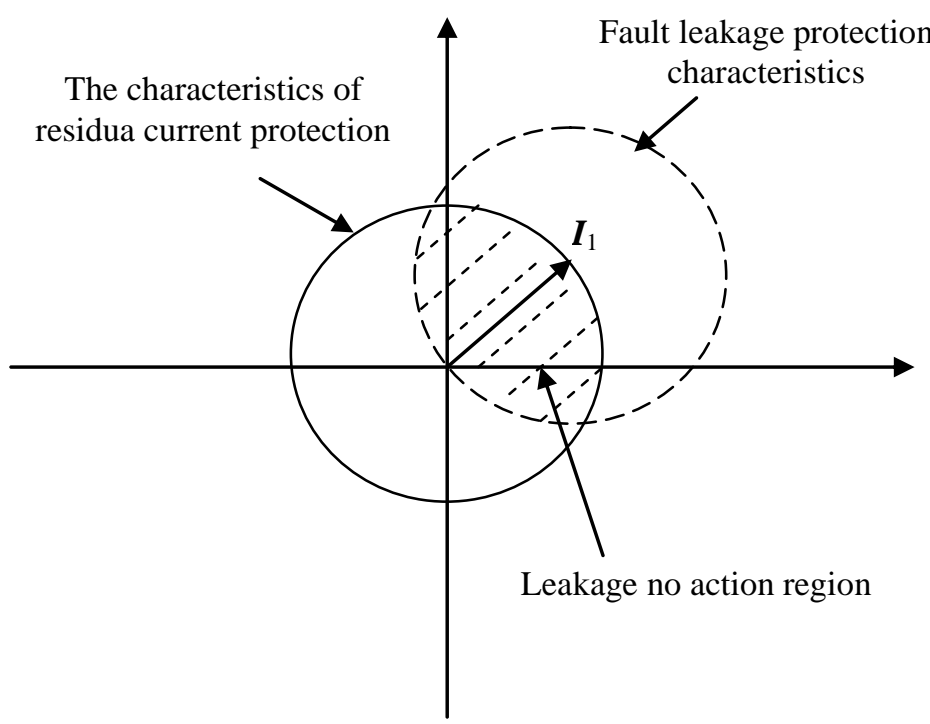

Fig. (4). Leakage current protection characteristic based on bi-criteria of the residual current and its variation.

constitutes the amplitude discrimination and phase discrimination leakage protection technology. Use of amplitude discrimination and phase discrimination technology of leakage protection for the protection of human electric shock, generally choose the smaller action value. Therefore, amplitude discrimination and phase discrimination technology of leakage protection malfunction can be easily produced, considering the condition of the power network and the need of equipment in use, the scope of use restrictions.

\subsection{Catastrophe Model of Leakage Protection}

In power system, fault leakage generated generally into two kinds of situations, one is that the mutation of leakage, leakage caused by sudden failure, sudden change of residual current, the other one is slow change leakage, namely fault is slowly changing, and residual current caused by the change is relatively slow. This is based on two conditions, can adopt different methods of detection and protection.

In order to fault detection of sudden, detected by continuous movement change method. Continuous movement change law is based on a Zhou Bo residual current detection unit, comparison of residual current and front of the fixed time interval each time the calculated mutation, leakage current of the time. In general, the leakage current will be $2 \mathrm{mu}-$ tations in 5 cycles to reach steady state, in this period of time, the leakage current is basically stable mutation. When choosing the detection interval time is 3 cycles, detection model for mutation leakage:

$\dot{I}_{\mathrm{A} \triangle i}=\dot{I}_{i} \quad \dot{I}_{i 3}$

Therefore, the detection and calculation, only need to store the I moment ago 3 residual current, i.e. $\dot{I_{i-3}}, \dot{I_{i-2}}, \dot{I_{i} 1}$, and does not need to be memorized all the historical data, reduce the storage capacity.

\subsection{Continuous Slow Change Leakage Protection Model}

Continue to slow change leakage, slow change leakage continues for a long time, affected by the memory capacity limitations, can not be slowly varying duration in all the residual current record. Methods of continuous slow change leakage protection implementation is: continuous detection for slow change leakage current by using moving window variation, as a Zhou Bo residual current detection unit, a comparison of the residual current and each time in front of the fixed window starting time, calculate the residual current changes in the moment of quantity, namely continuous slow change leakage current.

The actual corrosion rate of change increases the leakage current is unknown, and a large range. Leakage current detection and protection so as to different continuous slow variation in speed, while using multiple windows of time, for example: while using $1 \mathrm{~S}, 10 \mathrm{~S}, 60 \mathrm{~S}, 120 \mathrm{~S}, 180 \mathrm{~S}, 300 \mathrm{~S}$ as the window of time. In the power supply frequency is $50 \mathrm{~Hz}$, a weekly period detection data, the above window time can be discredited into 50, 500, 3000, 6000, 9000, 15000. Slow change leakage current detection model for:

$$
\begin{aligned}
& \dot{I}_{\mathrm{S} \Delta i}=\dot{I}_{i} \quad \dot{I}_{N T} \\
& (N+1) T>i \geqslant N T
\end{aligned}
$$

Residual current start time window type in the $\mathrm{N}$, said the moment in the $\mathrm{N}$ calculation window, $\mathrm{T}$ said the value of the time discrediting window.

Using formula (13) calculate the sustained slow change leakage current, residual current start time only need to record the I moments before the window, when using the 6 window of time, residual current starting time only record 6 window, can realize continuous slow change leakage protection. 


\section{TIME DELAY TYPE LEAKAGE PROTECTION METHOD BASED ON VARIATION LAW}

Delay is the basic requirement of leakage protection, leakage protection delay time is generally in the $0.2 \mathrm{~S}$ above, some require up to $0.5 \mathrm{~S}$ or more than $1 \mathrm{~S}$. The following analysis is providing to solve leakage variation leakage delay protection case problems in calculation.

\subsection{Mutation of Fault Delay Protection Method}

Fault current i moments occur more than action value, time $i+1, i+2, i+3, i+4$, i fault leakage current of $\mathrm{i}+4$ remains the same, fault current and i time of the same type (7), according to the calculation of residual current variation (i.e., fault current), variation i+4 moment for the 0 , and then the fault time only 4 Zhou Bo $(0.08 \mathrm{~S})$, unable to realize the time delay protection fault leakage. The need for protection method is improved. The improved method is: when the moment of i detected residual current variation reaches a certain value (such as leakage protection action value), by equation (20) of fault current calculation of fault duration within the $\mathrm{I}+\mathrm{k}$ moment.

$\dot{I}_{\Delta(i+k)}=\dot{I}_{i+k} \quad \dot{I}_{i 3}$

When the mutation of fault current exceeds the duration of the delay time, leakage protection; if the fault current mutation in delay time less than action value, then the type (14) residual current variation calculation is less than action value, re using formula (12) calculation of residual current variation

\subsection{Continued Slowly Varying Fault Leakage Delay Pro- tection Method}

Calculated using several different window duration of slowly varying fault leakage current, within each window according to the residual current start time calculation slow variables, but the occurrence time of slow change leakage current is not the start time window, at the same time, slow change leakage may span two windows. When detecting a constant slow change leakage, use the following method to realize effective protection.

1) When a constant slow to action value of leakage current change, automatically extended window time, namely the initial residual current calculation amount of change has been the same, to achieve accurate detection and delay constant slow change leakage, by formula (15) calculation.

$$
\dot{I}_{\mathrm{S} \Delta(i+k)}=\dot{I}_{(i+k)} \quad \dot{I}_{N T}
$$

2) When a constant slow exceeds a certain value in a time window leakage current variable (such as 0.3Id), automatically extend the time window, and maintain the three window of time. If continued to slow in the three windows of time action value does not reach the leakage current change, re determine the window start time.

3) Continued to slow in delay time than action value of fault current is changed, leakage protection; when a constant slow in delay time less than action value of fault current is changed, re determine the calculation of continuous slow window starting time varying fault leakage current.

Through the above methods, can be implemented on any moment of slow change leakage effectively delay protection.

\section{CONCLUSION}

This paper analyzes the leakage protection technology, put forward the principle of leakage protection of residual current based on vector data, and according to the characteristics of leakage current, is proposed to identify the fault of electric leakage and protection method. Through this thesis research, obtained the following conclusions:

(1) Through the analysis of the residual current protection technology, causes of leakage protection dead zone of residual current amplitude method based on the theoretically.

(2) Put forward fault identification technology, and analyzes the calculation method of residual current vector variation, triangle formulas residual current variation calculation method, orthogonal decomposition approach for calculating method and three-phase equivalent.

(3) Put forward the principle of leakage protection of residual current based on vector namely uses the residual current amplitude and residual current variation of leakage protection, can effectively eliminate the leakage protection dead zone, and guarantee the power system operation rate.

(4) According to the characteristics of fault leakage detection model, put forward mutation leakage and continuous slow change leakage two fault leakages, ensuring the reliability of fault leakage protection method and effectiveness.

(5) Put forward a delay residual current leakage protection method based on vector.

\section{CONFLICT OF INTEREST}

The authors confirm that this article content has no conflict of interest.

\section{ACKNOWLEDGEMENTS}

Declared none.

\section{REFERENCES}

[1] S. Teng, and X. Yang, Leakage Current Operated Protective Device and Its Application, China Machine Press, China 1994.

[2] A.L. Kidd, and G.H. Scholes, "Successful selection and operation of overcurrent and residual current protective measures," In: Proceedings of the International Conference on Installation Engineering Designing and Maintaining Successful Systems, 1998.

[3] K. Nomura, F. Hisalsune, and T. Yamada, "The present state and future trends in circuit breakers and earth leakage circuit breaker," Mitsubushi Denki Giho, vol. 69, no. 4, pp. 12-14, 1995.

[4] K. Li, J. Lu, and J. Qu, "Study on intelligent protective relay with discriminating phase and amplitude of residual current," Low Voltage Apparatus, no. 4, pp. 12-14, 2001.

[5] K. Li, and J. Lu, "Study on electromagnetic compatibility for intelligent residual current operated protective deice," Low Voltage Apparatus, no. 6, pp. 8-12, 2002. 
[6] K. Li, J. Lu, and J. Qu, "Study on the reliability compliance test for residual current operated protector," Transactions of china Electrotechnical Society, vol. 18, no. 4, pp. 114-118, 2003.

[7] X. Dai, "A brief discussion on the protection relay based on power frequency variation principle," Automation of Electric Power Systems, vol. 19, no. 1, pp. 41-47, 1995.

[8] B. Su, J. He, X. Dong, and Y. Zhang, "New scheme of high speed power line carrier protection based on power frequency voltages and currents," In: Proceedings of the CSU-EPSA, vol. 12, no. 6, pp. 37-40, 2000.

[9] Q. Huang, K. Shuang, P. Xu, and J. Li, "Prediction-based dynamic resource scheduling for virtualized cloud systems", Journal of Networks, vol. 9, no. 2, pp. 375-383, 2014.
[10] X. Hu, and L. Sun, " $S F_{6}$ Study on the method of circuit breaker on-line insulation monitoring", Electric power automation Equipment, vol. 26, no. 4, pp.253-258, 2006.

[11] Y. Wang, Z. Piao, X. Wang, and H. Liu, "Several calculation methods for the electrical life of on-line motoring circuit breakers", Journal of Shenyang Agricultural University, vol. 36, no. 2, pp. 7883, 2005.

[12] B. Li, "Value and its online monitoring technology conversion, the electrical life of circuit breakers limit", High Voltage Electrical Apparatus, vol. 41, no. 6, pp. 43-49, 2005.

[13] Y. Ni, and C. Hang, "Detection of underwater carrier-free pulse based on time-frequency analysis", Journal of Networks, vol. 8, no. 1 pp. 205-212, 2013.

(C) Zixing et al.; Licensee Bentham Open.

This is an open access article licensed under the terms of the Creative Commons Attribution Non-Commercial License (http://creativecommons.org/licenses/by$\mathrm{nc} / 3.0 /$ ) which permits unrestricted, non-commercial use, distribution and reproduction in any medium, provided the work is properly cited. 